\title{
Particle dispersion processes in two-dimensional turbulence: a comparison with 2-D kinematic simulation.
}

\author{
R. Castilla ${ }^{1}$, J. M. Redondo ${ }^{2}$, P. J. Gámez-Montero ${ }^{1}$, and A. Babiano ${ }^{3}$ \\ ${ }^{1}$ LABSON, Dpt. of Fluid Mechanics, U.P.C., Spain \\ ${ }^{2}$ Dpt. of Applied Physics, U.P.C., Spain \\ ${ }^{3}$ CNRS, Laboratoire de Météorologie Dinamique, Ecole Normale Superieur, France
}

Received: 8 January 2007 - Revised: 14 March 2007 - Accepted: 20 March 2007 - Published: 28 March 2007

\begin{abstract}
We study numerically the comparison between Lagrangian experiments on turbulent particle dispersion in 2-D turbulent flows performed, on the one hand, on the basis of direct numerical simulations (DNS) and, on the other hand, using kinematic simulations (KS). Eulerian space-time structure of both DNS and KS dynamics are not comparable, mostly due to the absence of strong coherent vortices and advection processes in the KS fields. The comparison allows to refine past studies about the contribution of nonhomogeneous space-time 2-D Eulerian structure on the turbulent absolute and relative particle dispersion processes. We particularly focus our discussion on the Richardson's regime for relative dispersion.
\end{abstract}

\section{Introduction}

Many turbulent flows in geophysics can be studied using 2$\mathrm{D}$ barotropic models. One important characteristic of the two-dimensional turbulence is the capacity to self-organize into strong large-scale coherent vortices which produce a complex non-homogeneous topology in the physical space (McWilliams, 1984). Direct Numerical Simulations (DNS) show that two-dimensional fields may be described in term of an elementary partitioning of both vorticity and strain (Okubo et al., 1970; Weiss, 1991; Elhmaïdi et al., 1993). The relative dominance of one over the other allows us to distinguish elliptic domains (high vorticity concentrations inside vortices) and hyperbolic ones (deformation cells on vortex periphery which are characterized by high strain and turbulent energy). The residual field composed of moderate elliptic/hyperbolic regions is considered as a quasihomogeneous turbulent background. Inhomogeneities in the physical space and the long-range effect of coherent vor-

Correspondence to: R. Castilla

(castilla@mf.upc.edu) tices have a significant influence on the particle trajectories and on the Lagrangian statistical analyses (Pasquero et al., 2001). The whole problem in the physical investigation of these flows is the recognition of both Eulerian flow's structure and diffusion properties from Lagrangian data involving absolute and relative particle dispersion measurements (Ollitraut and Colin de Verdière, 2002a,b; LaCasce and Bower, 2000; Provenzale, 1999).

An important question in the framework of general theories of turbulence is to clarify the reason which can support the consistency between large-scale vortex contributions and similarity predictions for relative dispersion. Richardson's scaling-law for relative dispersion is usually considered as a robust link between particle separation and homogeneously developed turbulent cascade of energy (Richardson, 1926; Obukhov, 1941). However, numerical Lagrangian experiments on two-dimensional turbulence indicated that Richardson's regime can be more than that since it is often sufficiently well observed even when the inverse energy cascade is not completely achieved (Zouari and Babiano, 1994). This fact creates an apparent inconsistency in our understanding of the Richardson's regime in two-dimensional turbulence. On the other hand, Elhmaïdi et al. (1993) have observed a recurrent anomalous behaviour for absolute dispersion in $t^{5 / 4}$ between the ballistic regime at small dispersion times and the Brownian asymptotical behaviour for long times. At present, even if we still do not have a conclusive certitude founded on Lagrangian measurements, the assumption is that this anomalous behaviour constitutes a complementary example of the direct non-homogeneous contribution of coherent vortices.

The objective of the present paper is to illustrate the above problems and to investigate the contribution of the nonhomogeneous 2-D space-time structure to the dispersion processes. We compare absolute and relative dispersion produced, on the one hand, by the DNS Eulerian space-time structure and, on the other hand, by the Lagrangian stirring

Published by Copernicus GmbH on behalf of the European Geosciences Union and the American Geophysical Union. 
induced by a Kinematic Simulation (KS) (Kraichnan, 1966; Malik and Vassilicos, 1999). Kinematic Simulation used represents a pseudo-turbulent field which in our numerical investigation will be characterized by the same spectral features as DNS field. Since KS do not represent a temporal evolution solution, the main contributions to the Lagrangian dynamics of typical 2-D space-time structures namely, coherent vortices and advection processes are smoothed or disappeared (Pasquero et al., 2001). Even when the two flows are characterized by equivalent Eulerian energy spectra, the difference between DNS and KS dynamics lies in different temporal evolutions from both Eulerian and Lagrangian point of views. Consequently, our study does not allow to distinguish unambiguously and properly the direct contribution of every important components of the 2-D Eulerian dynamics. However, the signature of the coherent vortices as organizing centres of the space-time evolution of the DNS dynamics compared to the KS Lagrangian stirring one is evident enough in some cases.

We will compare Lagrangian-KS results with previously performed Lagrangian-DNS investigations (Babiano et al., 1987; Babiano and Le Roy, 1987; Elhmaïdi et al., 1993; Babiano et al., 1990; Zouari and Babiano, 1994). A clear observation is that the intermediate $t^{5 / 4}$ anomalous absolute dispersion law and the Richardson's $4 / 3$ scale-regime for relative dispersion seem to be reinforced due to the presence of the coherent vortices. In all the cases, KS Lagrangian experiments show hyperdiffusion properties compared with the behavior of DNS simulations of 2-D flows.

The paper is organized as follows. In Sect. 2, general theories of absolute and relative particle dispersion are presented. In Sect. 3 both DNS and KS numerical approaches are briefly described and basic dynamical properties of both simulations are discussed. Results concerning our KS-Lagrangian experiments are presented in Sect. 4. The final discussion and the conclusions are detailed in Sect. 5.

\section{Definitions: absolute and relative dispersion}

We consider the evolution of the separation of neutral fluid particle pairs which, initially separated by a given distance, are advected by a two-dimensional Eulerian velocity field $\mathbf{u}(\mathbf{x}, t)$. For every pair of particles with Lagrangian coordinates $\mathbf{a}_{1}$ and $\mathbf{a}_{2}$, the relative separation vector $\boldsymbol{D}$ at time $t$ is related by the initial separation vector $\boldsymbol{D}_{0}=\mathbf{a}_{1}-\mathbf{a}_{2}$ and the absolute displacement vector $\mathbf{A}$ by

$\mathbf{D}\left(t, \mathbf{D}_{0}\right)=\mathbf{D}_{0}+\left[\mathbf{A}\left(\mathbf{a}_{1}, t\right)-\mathbf{A}\left(\mathbf{a}_{2}, t\right)\right]$.

The absolute displacement vector $\mathbf{A}$ is defined in term of the Lagrangian velocity $\mathbf{v}(\mathbf{a}, t)=\mathbf{u}(\mathbf{x}(\mathbf{a}, t), t)$ as

$\mathbf{A}(\mathbf{a}, t)=\int_{0}^{t} \mathbf{v}(\mathbf{a}, \tau) \mathrm{d} \tau$
From Eq. (1) and the definitions of the relative Lagrangian velocity $\delta \mathbf{v}=d \mathbf{D} / d t$ and the Lagrangian relative acceleration $\delta \boldsymbol{\Gamma}=d \delta \mathbf{v} / d t$, one can write:

$\mathbf{D}\left(t, \mathbf{D}_{0}\right)=\mathbf{D}_{0}+\int_{0}^{t} \delta \mathbf{v}\left(\tau, \mathbf{D}_{0}\right) \mathrm{d} \tau$,

where

$\delta \mathbf{v}\left(t, \mathbf{D}_{0}\right)=\delta \mathbf{v}_{0}+\int_{0}^{t} \delta \boldsymbol{\Gamma}\left(\tau, \mathbf{D}_{0}\right) \mathrm{d} \tau$.

Using Eqs. (3) and (4), the time evolution of both absolute and relative dispersion efficiencies $K(t)$ and $Y(t)$ (dispersion coefficients) are given, respectively, by

$K(t)=\frac{1}{2} \frac{\mathrm{d} A^{2}}{\mathrm{~d} t}=<\mathbf{A}(\mathbf{a}, t) . \mathbf{v}(\mathbf{a}, t)>_{\mathbf{a}}$

and

$Y\left(t, D_{0}\right)=\frac{1}{2} \frac{\mathrm{d} D^{2}}{\mathrm{~d} t}=<\mathbf{D}\left(t, \mathbf{D}_{0}\right) . \delta \mathbf{v}\left(t, \mathbf{D}_{0}\right)>$,

where $<_{.}>_{\mathbf{a}}$ refers to the average at time $t$ over all particles a and $<$.> refers to the average at time $t$ over all particle pairs initially separated by a given distance $D_{0}=\left\|\mathbf{D}_{0}\right\|$. From Eqs. (5) and (2),

$K(t)=\int_{0}^{t}<\mathbf{v}(\mathbf{a}, t) \cdot \mathbf{v}(\mathbf{a}, \tau)>_{\mathbf{a}} \mathrm{d} \tau$.

Similarly, from (6) and (3),

$$
\begin{array}{r}
Y(t)=2 K(t)-2 \int_{0}^{t}<\mathbf{v}\left(\mathbf{a}_{1}, t\right) . \mathbf{v}\left(\mathbf{a}_{2}, \tau\right)>_{\mathbf{a}} \mathrm{d} \tau \\
-<\mathbf{D}_{0} . \delta \mathbf{v}\left(t, \mathbf{D}_{0}\right)>,
\end{array}
$$

where the last term vanishes if the dispersion process is statistically homogeneous.

\subsection{Absolute dispersion}

The starting point of the kinematic analysis of the absolute dispersion is based on the rigorous definition (7) which, in terms of the second-order Lagrangian velocity structure function $S_{L}(\tau)=\frac{1}{2}<\|\mathbf{v}(\mathbf{a}, t)-\mathbf{v}(\mathbf{a}, t+\tau) \quad\|^{2}>_{a}$ can be rewritten as

$K(t)=2 E \int_{0}^{t}\left(1-\frac{S_{L}(\tau)}{2 E}\right) \mathrm{d} \tau$,

where $E=1 / 4<\left\|\mathbf{v}\left(t_{0}\right)\right\|^{2}+\left\|\mathbf{v}\left(t_{0}+t\right)\right\|^{2}>_{\mathbf{a}, t}$ refers to the Lagrangian turbulent energy along particle trajectories. The asymptotic behaviour of $S_{L}(\tau)$ at small times is given after a Taylor's expansion: $S_{L}(t) \sim C t^{2}$, where $C$ is a dimensional constant. In a statistically steady incompressible turbulence the constant $C$ can be approximated by the gradient of the 
pressure forces: $C=\|\nabla P\|^{2}$. Under stationarity assumption and provided that the Lagrangian structure function converges at long dispersion times toward twice the Lagrangian energy $E$, one classically obtains the following asymptotic behaviours for the absolute particle dispersion (Taylor, 1921; Kampé de Fériet, 1939; Babiano and Le Roy, 1987):

$A^{2}(t) \sim 2 E t^{2}\left(1-\frac{C}{12 E} t^{2}\right)$,

$K(t) \sim 2 E t\left(1-\frac{C}{6 E} t^{2}\right)$,

for small dispersion times, and

$A^{2}(t) \sim 2 K t$,

$K \sim 2 E T_{L}$,

for long dispersion times. The validity of Eqs. (10-11) is commonly accepted for $t<T_{L}$, where $T_{L}$ is the Lagrangian integral timescale defined as the saturation value of the integral

$T_{L}=\lim _{t \rightarrow \infty}(2 E)^{-1} \int_{0}^{t}<\mathbf{v}(\mathbf{a}, t) \cdot \mathbf{v}(\mathbf{a}, \tau)>_{\mathbf{a}} \mathrm{d} \tau$.

Thus, the kinematic approach of statistically homogeneous and stationary absolute dispersion is based on the rigorous relation between the variance $A^{2}(t)$ and the second-order Lagrangian velocity structure function $S_{L}(\tau)$. Numerical investigation performed by Elhmaïdi et al. (1993) have shown that inhomogeneities in the Eulerian velocity field generate an anomalous $t^{5 / 4}$ law of the absolute particle dispersion between both the ballistic Eq. (10) and the Brownian Eq. (12) asymptotic behaviours. It seems worthwhile to investigate if $\mathrm{KS}$ and its corresponding Lagrangian dynamics produces the same anomalies in the absolute dispersion behaviour as DNS at intermediate times.

\subsection{Relative dispersion}

The assumption about the stationarity in the absolute dispersion processes is correct if the turbulence is really statistically stationary i.e., the absolute dispersion is invariant under a change of the initial time. The situation is quite different for relative dispersion processes: even for a stationary turbulence, the relative dispersion is generically non stationary since all its functional characteristics depend on the time-lag $\tau$ in addition to the separation scale $D(t)=\left\|\mathbf{D}\left(t, \mathbf{D}_{0}\right)\right\|$ (Batchelor, 1952).

The Lagrangian phenomenological simplification of the exact relation (6) is based on the dimensional argument:

$Y(D) \sim S(D)^{1 / 2} D$, where $D=<D^{2}>^{1 / 2}$ is the root-mean-square pair's separation at time $t$ and $S(D)$ refers to the second-order Eulerian velocity structure function at separation $D$. Since $S(D)$ and the Eulerian energy spectrum $E(k)$ are linked by

$S(D)=4 \int \sin ^{2} \frac{k D}{2} E(k) \mathrm{d} k$,

then, from Eqs. (15) and (16) we obtain the following qualitative behaviours at intermediate length-scales for a powerlaw Eulerian energy spectrum $E(k) \sim k^{-m}$ (Larchevêque and Lesieur, 1981; Lesieur, 1985; Bennet, 1987; Babiano et al., 1990):

$$
\begin{aligned}
1<m<3, \quad S(D) \sim D^{m-1}, Y(D) & \sim D^{(m+1) / 2} ; \\
m \geq 3, \quad S(D) & \sim D^{2}, \quad Y(D) \sim D^{2} .
\end{aligned}
$$

Within the limits of the Kolmogorov-Kraichnan theory, the self-similar prediction in the energy inertial range provides $m \approx 5 / 3$. Thus, this covers, as a particular case, Richardson's 1926 dispersion law for $S(D) \sim D^{2 / 3}(m \approx 5 / 3)$ which would apply in dimension two to the inverse energy cascade:

$Y(D) \sim D^{4 / 3}$

or, integrating over time $t$

$D^{2} \sim t^{3}$.

If we define, as in Babiano et al. (1990), the relative dispersion characteristic time $\tau_{R}$ as

$\tau_{R}(D)=\frac{D^{2}}{Y(D)}$,

then, phenomenological relations (19) and (20) reduce to

$\tau_{R}(D) \sim D^{2 / 3}$.

We may consider an alternative approach to deduce the Richardson's time-regime (20). Actually, from Eq. (3) we can deduce the differential equation which governs the time evolution of the relative separation variance. This equation may be expressed as

$\frac{\mathrm{d}}{\mathrm{d} t} \frac{D^{2}-D_{0}^{2}}{t}=\left\|\delta \mathbf{v}\left(\mathbf{D}_{0}, t\right)\right\|^{2}-\left\|\frac{1}{t} \int_{0}^{t} \tau \delta \boldsymbol{\Gamma}(\tau) \mathrm{d} \tau\right\|^{2}$,

(see Babiano et al., 1990, for a detailed discussion). The Eq. (23) is linear and the solution for the averaged set of particle pairs initially separated by a given distance $D_{0}$ is given by

$<\left\|D-D_{0}\right\|^{2}>=t \int_{0}^{t} F\left(\tau, D_{0}\right) \mathrm{d} \tau$,

where

$F\left(t, D_{0}\right)=<\left\|\delta \mathbf{v}\left(t, D_{0}\right)\right\|^{2}>-G\left(t, D_{0}\right)$, 
$G\left(t, D_{0}\right)=<\left\|\frac{1}{t} \int_{0}^{t} \tau \delta \boldsymbol{\Gamma}\left(\tau, D_{0}\right) \mathrm{d} \tau\right\|^{2}>$.

If the correlations of Lagrangian relative accelerations

$R_{\delta \Gamma}\left(t, t-\sigma, D_{0}\right)=<\delta \boldsymbol{\Gamma}\left(t, \mathbf{D}_{0}\right) . \delta \boldsymbol{\Gamma}\left(t-\sigma, \mathbf{D}_{0}\right)>$

are statistically stationary, i.e. they depend on the time lag $\sigma=t-\tau$ but not on the dispersion time $t$, then the analytical development of the solution (24) in the stationary and homogeneous approximation yields (Babiano et al., 1990; Zouari and Babiano, 1994; Lin and Reid, 1963; Monin and Yaglom, 1975)

$D^{2}\left(t, D_{0}\right)=D_{0}^{2}+\left(\left\|\delta \mathbf{v}_{\mathbf{0}}\right\|^{2}+\left.G(t)\right|_{\text {stationary }}\right) t^{2}$,

where

$\left.G(t)\right|_{\text {stationary }}=\frac{2}{3} I(t) t\left(1-\frac{3}{2} \frac{o(t)}{t}\right)$,

$I(t)=\int_{0}^{t} R_{\delta \Gamma}\left(t, t-\sigma, D_{0}\right) \mathrm{d} \sigma$.

Here $\left.G(t)\right|_{\text {stationary }}$ refers to the function $G(t)$, defined as Eq. (26), expressed under the stationarity assumption (for simplicity we have omitted on the left of Eqs. (29-30) reference to the dependency on $D_{0}$ ). Thus, the already assumed stationarity of $R_{\delta \Gamma}\left(t, t-\sigma, D_{0}\right)$ ensures the saturation of the integral $I(t)$ in the right-hand side of Eq. (29). This recovers from Eq. (28) the Richardson's time-regime (Eq. 20).

The physical meaning of the stationarity assumption is that $R_{\delta \Gamma}\left(t, t-\sigma, D_{0}\right)$ no longer depends on the amplitude of separation $D$ at both times $t$ and $t-\sigma$ but only depends on the time-lag $\sigma$. The experimental verification of this property is conceivable only in the framework of numerical experiments when the Lagrangian acceleration field is determined with sufficiently enough accuracy.

Using the stationarity assumption we also get from Eq. (23) the following simplified equation:

$$
\left.\frac{\mathrm{d}}{\mathrm{d} t} D^{2}\right|_{\text {stationary }}=\left(<\left\|\delta \mathbf{v}_{\mathbf{0}}\right\|^{2}>+<\left\|\delta \mathbf{v}\left(\mathbf{D}_{\mathbf{0}}, t\right)\right\|^{2}>\right) t .
$$

If we define the ratio

$$
\Phi(t)=\frac{\left.\frac{\mathrm{d}}{\mathrm{d} t} D^{2}\right|_{\text {observed }}}{\left.\frac{\mathrm{d}}{\mathrm{d} t} D^{2}\right|_{\text {stationary }}},
$$

then, the domain of validity of the stationarity assumption will be determined by the time interval in which $\Phi(t)$ exhibits a plateau. The consistency between Eqs. (28) and (31) is guaranteed by the linear growth in time of $<\left\|\delta \mathbf{v}\left(\mathbf{D}_{\mathbf{0}}, t\right)\right\|^{2}>$ in the stationary relative dispersion regime (Babiano and Le Roy, 1987; Babiano et al., 1990). Relation (32) have the advantage over the analysis based on Eqs. (27) and (28) that all terms are accessible to measurements.

To summarize, there are two parallel arguments, independent from each other, that predict the Richardson's dispersion regimes (19-20). A phenomenologic approach and a kinematic one (Zouari and Babiano, 1994). The quasi-stationary regime at large separation length-scales is probably always reached if the initial pair separation is small enough. The important question is to determine how far the action on the turbulent Lagrangian advection of the 2-D space-time structure favours the establishment of such a stationary regime. For this reason, it seems worthwhile to investigate how expressions (30) and (32) behave with respect to the KS Lagrangian stirring.

The prediction is that the saturation of the contribution of non-stationary terms in the Eq. (23) is favoured by the action of coherent vortices in DNS dynamics.

\section{Numerical simulations}

\subsection{Direct Numerical Simulation (DNS)}

We will consider different steady Eulerian-DNS with different resolutions and configurations already analyzed in the Lagrangian framework by Babiano et al. (1987, 1990), Elhmaïdi et al. (1993) and Zouari and Babiano (1994). All these simulations obviously show a high prominence of coherent structures of different typical sizes which lie in the forcing wavenumber $k_{I}$. They are obtained by solving the 2$\mathrm{D}$ vorticity equation with forcing and dissipative terms using standard pseudospectral approximation in a double periodic domain of size $2 \pi \times 2 \pi$ :

$$
\frac{\partial}{\partial t} \omega+J(\psi, \omega)=f(\omega)+g(\omega) .
$$

Here $\omega$ is the vorticity, $\psi$ is the stream function, $J(\cdot, \cdot)$ refers to the two-dimensional Jacobian, $f(\omega)$ and $g(\omega)$ denote sources and sinks respectively. The forcing $f(\omega)$ is defined by keeping constant the energy density at a given wavenumber $k_{I}$. The sink $g(\omega)$ is the sum of a large scale linear friction and a small scale dissipation. The latter is defined by a 8-order iterated Laplacian or by the "anticipated potential vorticity method" (Sadourny and Basdevant, 1985). The Lagrangian motion of the fluid particles was obtained using a second order time scheme and a third order spline interpolation.

Numerical simulations usually yield strong slope of the energy spectra in the direct enstrophy cascade compared to the self-similar prediction in $k^{-3}$. This is the direct effect of coherent structures and localness in the physical space (Babiano et al., 1985). However, the inverse energy cascade has local properties and is thus characterized by spectra which 
satisfy the $k^{-5 / 3}$ self-similar prediction. Even if the spectral signature of the coherent vortices is absent in the repartition of the energy density in the inverse energy cascade, both inverse energy transfers and large-scale vortices have a fundamental significance for the turbulent advection and dispersion in the physical space. This fact indicates that selfsimilarity results and phenomenological descriptions of dispersion processes at large scales in two-dimensional turbulence seems to be quite weak and deserve a careful analysis.

\subsection{Kinematic Simulation (KS)}

KS is a gridless model widely described in Fung (1990). This model was first proposed by Kraichnan $(1966,1970)$ and further developed and used by Fung et al. (1992); Fung and Vassilicos (1998), Castilla (2001) and references therein. For our purpose, its main characteristic is that it allows to choose the turbulent energy spectrum of the field. The Eulerian velocity field is defined as

$\mathbf{u}(\mathbf{x}, t)=\sum_{n=1}^{N}\left\{\mathbf{c}_{n} \sin \phi_{n}+\mathbf{d}_{n} \cos \phi_{n}\right\}$,

with

$\phi_{n}=\mathbf{k}_{n} \cdot \mathbf{x}+v_{n} t$

$\mathbf{c}_{n}$ and $\mathbf{d}_{n}$ are random direction vectors. Their magnitudes are related to the energy spectrum as

$\left\|\mathbf{c}_{n}\right\|^{2}=\left\|\mathbf{d}_{n}\right\|^{2}=\int_{k_{n}-1 / 2 \delta k}^{k_{n}+1 / 2 \delta k} E(k) \mathrm{d} k$.

The wavenumber vectors $\mathbf{k}_{n}$ have a random direction normal to both $\mathbf{c}_{n}$ and $\mathbf{d}_{n}$ in order to force the continuity. Then, fluid acceleration is given by

$\boldsymbol{\Gamma}(\mathbf{x}, t)=\sum_{n=1}^{N} v_{n}\left\{\mathbf{c}_{n} \cos \phi_{n}-\mathbf{d}_{n} \sin \phi_{n}\right\}$.

The time evolution of the field is governed by $v_{n}$, where $n=1, \ldots, N$ refer to eddies' turnover frequencies. Following Fung et al. (1992) and Fung and Vassilicos (1998), these frequencies are calculated as

$v_{n}=\lambda k_{n}^{3 / 2} E\left(k_{n}\right)^{1 / 2}$,

where $\lambda$ is a positive parameter which characterizes the steadiness of the mode $n$. For $\lambda=0$, we have a turbulent flow with "frozen" eddies. If $\lambda$ tends to infinity, the flow is completely unsteady. The value of $\lambda$ is determined here to be such that gives a similar Lagrangian time integral scale than for the DNS numerical experiments.

If we consider a power energy spectrum $E(k) \sim k^{-m}$, the Eq. (38) gives an increasing value of the turnover frequency $v_{n}$ with $n$ for $m<3$. For $m=5 / 3$, which corresponds to both the Komogorov's turbulence and the self-similar prediction $\delta u \sim l^{1 / 3}$, where $\delta u$ refers to the characteristic velocity of eddies of scale $l$, the turnover frequency behaves as $v_{n} \sim k_{n}^{2 / 3}$. For $m \geq 3$, the characteristic velocity is linear in $l$ (see Babiano et al., 1985) and, consequently, the turnover frequency should be constant with the scale. In our experiments we have maintained the expression (38), but it does not seem to affect the Lagrangian statistics.

In order to define KS initial conditions, we will consider energy spectra of already mentioned DNS Lagrangian experiments. Results on particle dispersion processes in these steady KS Eulerian regimes, where turbulent energy and enstrophy are constant and well stabilized in time, will be then compared with the results previously discussed.

\subsection{Comparison of models}

Energy spectra for DNS fields namely, R128F10 (with $128 \times 128$ grid resolution and forced at the wavenumber $k_{I}=10$ ), R512F40 (with $512 \times 512$ grid resolution forced at the wavenumber $k_{I}=40$ ) and R1024F256 (with $1024 \times 1204$ grid resolution and forced at wavenumber $k_{I}=256$ ) already used in Babiano et al. (1987, 1990), Zouari and Babiano (1994) and Elhmaïdi et al. (1993) are displayed in Fig. 1 as a function of the wavenumber $k$. DNS spectrum for R128F10 shows that the dynamics is dominated by a direct enstrophy cascade from the forcing wavenumber towards smaller scales. In addition, the inverse cascade of energy towards large scales is almost inappreciable. In the simulation R512F40, both direct enstrophy and inverse energy cascades are well developed. In both R128F10 and R512F40 simulations the spectral slope in the enstrophy range is close to -4 which is an indicator of the localness of the dynamics in the enstrophy cascade range. In the third simulation R1024F256, forced at higher wavenumber $k_{I}=256$, the direct enstrophy cascade is not well developed and the dynamics is dominated by an inverse cascade of energy which shows a theoretically consistent $-5 / 3$ spectral slope. KS spectra are not produced and represent in fact a steady input-constraint for the KS simulation. As illustration of similarities and differences between DNS and KS field's structure we focus here on the lower resolution R128F10 simulation.

KS and DNS vorticity fields are shown in Fig. 2. We observe the predominance of well structured vortices in the DNS field whereas the vorticity distribution is rather random in the KS one. Prominent vortex-structures in DNS fields have characteristic sizes of the order of forcing scale $D_{I}=\pi / k_{I}$. We will characterize the relative importance of the vortex size on the large-scale dynamics by the ratio between the typical vortex size $D_{I}$ and the box size $L$ as $\Re_{v}=D_{I} / L=\frac{1}{2 k_{I}}$. In the present case $\Re_{v}=0.05$ for DNS. Even if the typical value of the ratio $\Re_{v}$ is conspicuously less clear defined in KS field, it seems to be lower. For an equivalent distribution of the spectral energy density, the dynamics 

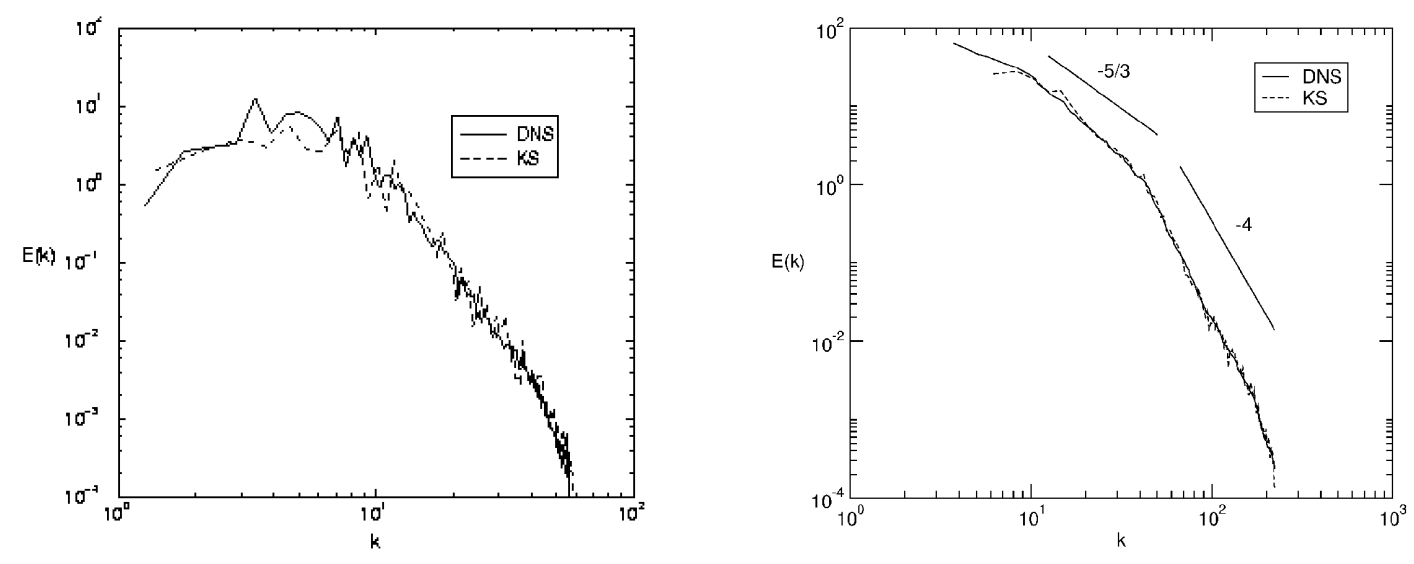

(a)

(b)

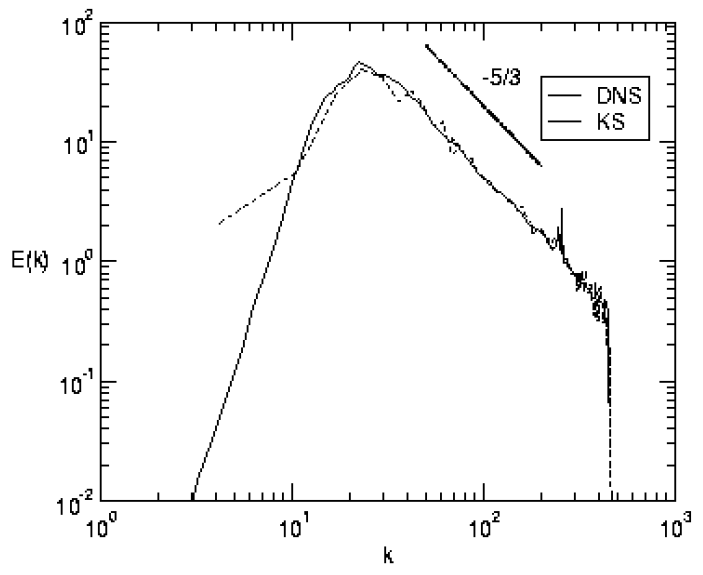

(c)

Fig. 1. Energy spectra as a function of the wavenumber $k$ for numerical experiments: (a) R128F10, (b) R512F40 and (c) 1024F256; DNS (continuous lines) and KS (dashed lines).

at large-scale seems to be less structured in KS rather than in DNS .

Figure 3 shows the distribution of the normalized OkuboWeiss parameter $Q(x)=\left(s^{2}-\omega^{2}\right) /\left(s^{2}+\omega^{2}\right)$ where $\omega$ and $s$ are the vorticity and the strain respectively (i.e. the antisymmetric and the symmetric parts of the velocity gradient tensor respectively (Okubo et al., 1970; Weiss, 1991)) and $x$ refers to the position in the physical space. Hyperbolic domains $(Q>0)$ characterize the high deformation cells which surround vortices. Elliptic domains $(Q<0)$, especially identify vorticity concentrations in the cores of cyclonic or anticyclonic vortices. Unlike the DNS field, the KS one shows a mixed topology where $Q$ is uniformly distributed and there are not clear structures.

In order to compare DNS and KS Lagrangian experiments we calibrated the two numerical simulations through the Lagrangian time-scale $T_{L}$ defined in Eq. (14). This time-scale typically lies in the Lagrangian stirring. We noticed that in the KS it depends on the steadiness factor $\lambda$ as Eq. (38). The ratio between the value of $T_{L}$ measured in DNS and different estimated values in KS are shown in Fig. 4 as a function of the steadiness factor $\lambda$. We can see that a sufficiently good consistency is reached for $\lambda \approx 0.5$. For $\lambda>1$, this ratio decreases. This means that for such a values of $\lambda$ the KS Lagrangian stirring exceeds the DNS one. According to this preliminary study, one may take $\lambda=0.5$. It is important to notice that, even for $\lambda=0.5$, the Eulerian time-scales in DNS and KS fields are very different since KS do not capture the Eulerian time evolution of the turbulent system (results not shown). Nevertheless, autocorrelation functions of the Lagrangian velocities present in the definition (14) are comparable to that observed between DNS and a stochastic model characterized by a bi-Gaussian Lagrangian velocity probability distribution function (see Pasquero et al., 2001). 


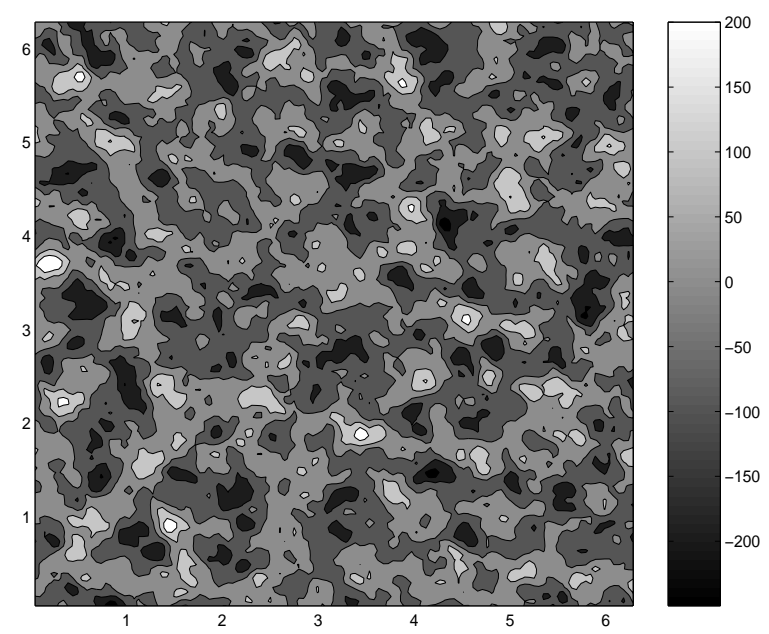

(a)

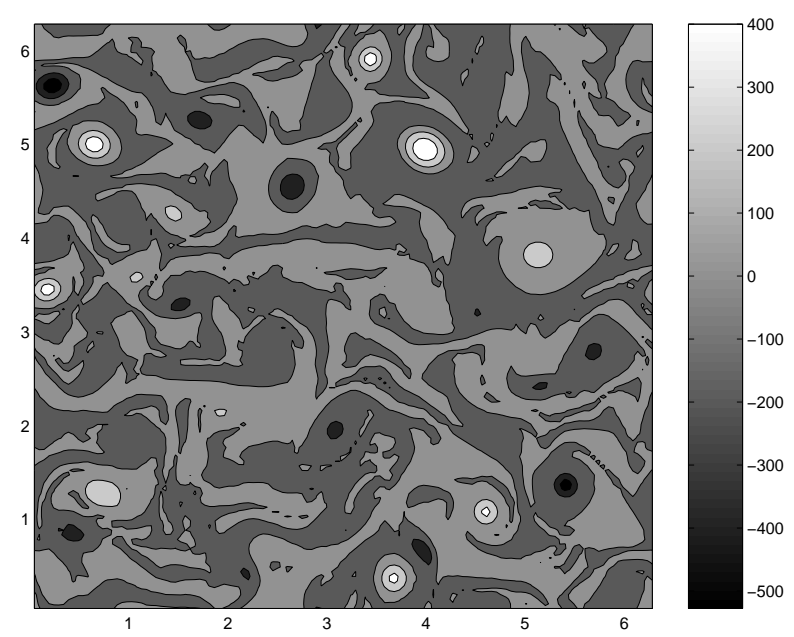

(b)

Fig. 2. Vorticity fields for R128F10: (a) KS, (b) DNS.

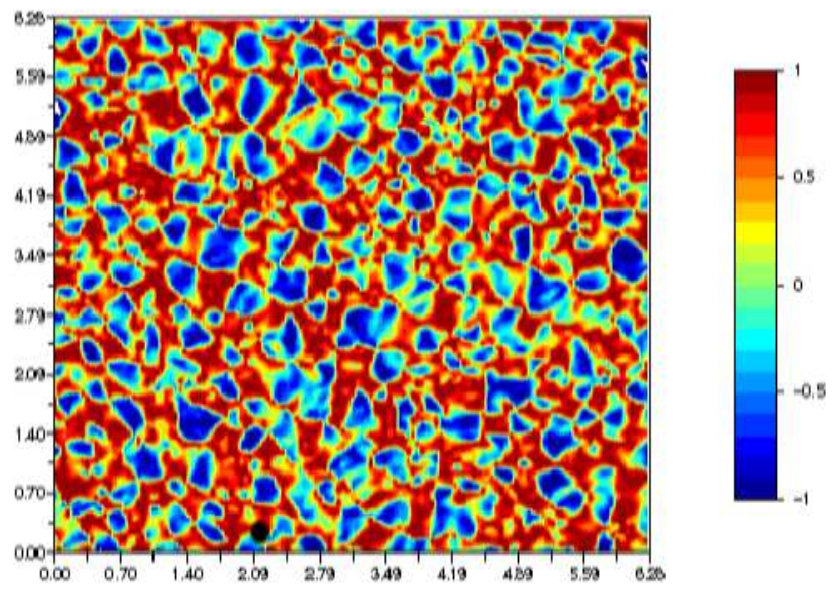

(a)

Fig. 3. Distribution of Q for experiment R128F10: (a) KS, (b) DNS.

\section{Numerical results}

\subsection{Absolute particle dispersion}

In this section we compare DNS and KS Lagrangian experiments using the field R128F10 that we have previously described. Our choice is motivated by the need to keep consistency with previous work (Elhmaïdi et al., 1993). Coherent structures in this field have quite an important size compared to the entire flow domain size $\left(\Re_{v}=0.05\right)$.

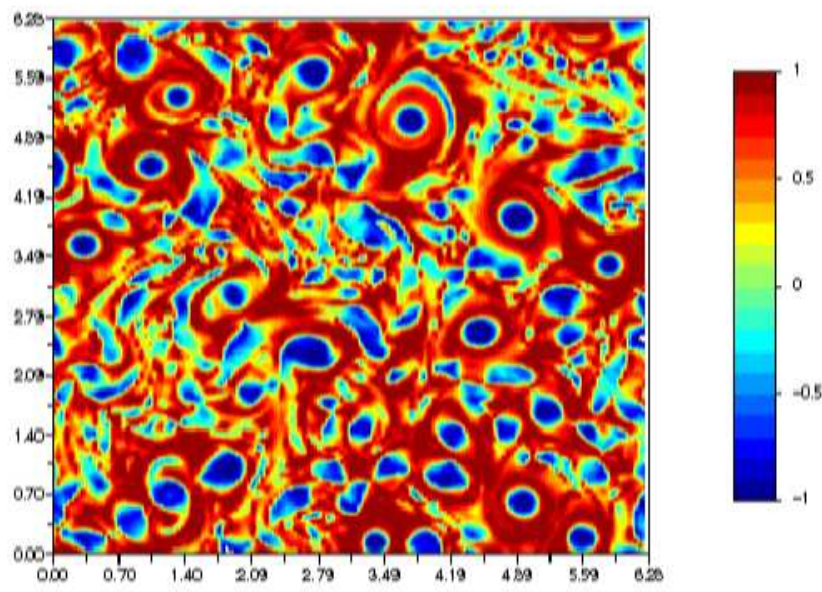

(b)

Figure 5 shows the absolute particle dispersion $A^{2}$ as a function of the normalized dispersion time $t / T_{L}$ for DNS (continuous line) and KS (dashed line). The two dispersion processes exhibit quite similar ballistic behaviour at small times. At intermediate times $\left(t \approx T_{L}\right)$, some difference is observed. After the ballistic regime (10), KS absolute dispersion shows an unscaled transient behaviour until the Brownian linear in time growth (12). This asymptotic behaviour is attained for dispersion times of the order of $t \approx 10 T_{L}$. DNS absolute dispersion, however, it clearly exhibits a well 


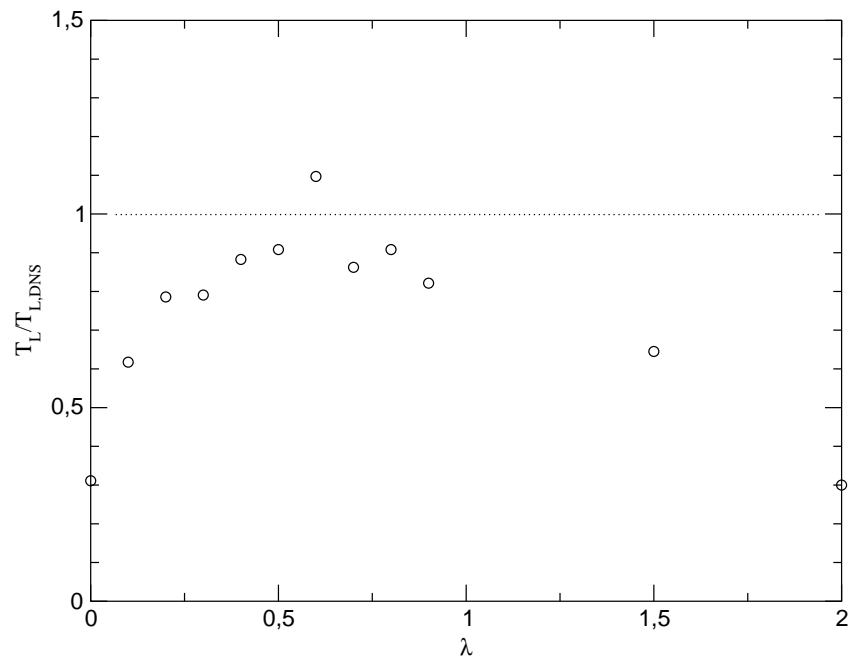

Fig. 4. KS Lagrangian time-scale as a function of $\lambda$.

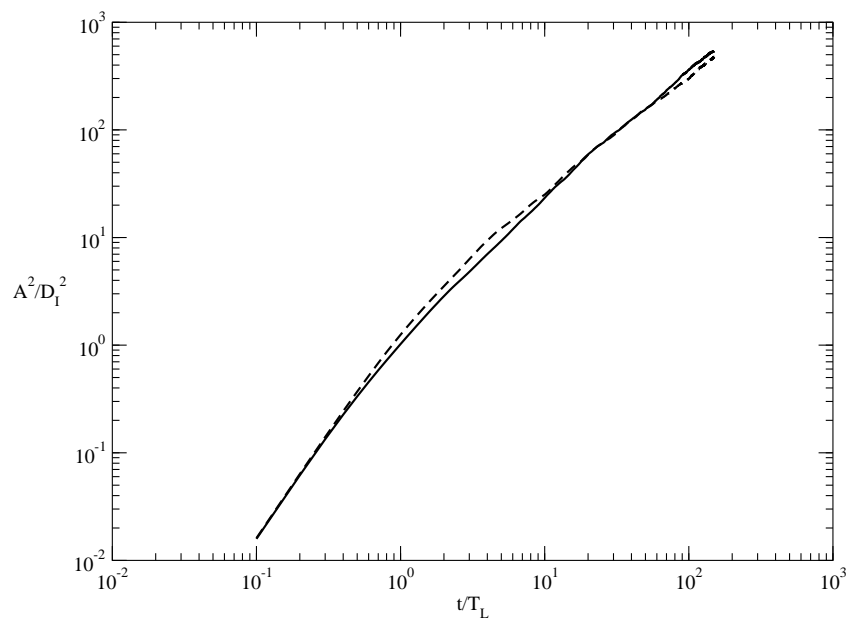

Fig. 5. Absolute dispersion $A^{2}$ as a function of time for R128F10 experiment: DNS (continuous line) and KS (dashed line).

defined scaling behaviour for a long time after $t \approx T_{L}$. To distinguish the local growth we compensated absolute dispersion by $t$ (fine lines) and $t^{5 / 4}$ (bold lines) in Fig. 6. Obviously, when the KS absolute dispersion shows a Brownian regime (fine dashed line) the DNS absolute dispersion behaves as $t^{5 / 4}$ (bold continuous line).

Elhmaïdi et al. (1993) argued that the $t^{5 / 4}$ anomalous regime is highly connected to energetic hyperbolic domains surrounding coherent vortices. The argument is that different domains of the Eulerian DNS field are not equally sampled by the Lagrangian dynamics. Neutral particles advected inside the vortices tend to leave internal-elliptic regions through a vorticity filamentation process. At the same time, particles advected on the external hyperbolic regions $(Q>0)$ surrounding vortices cannot penetrate into the exist-

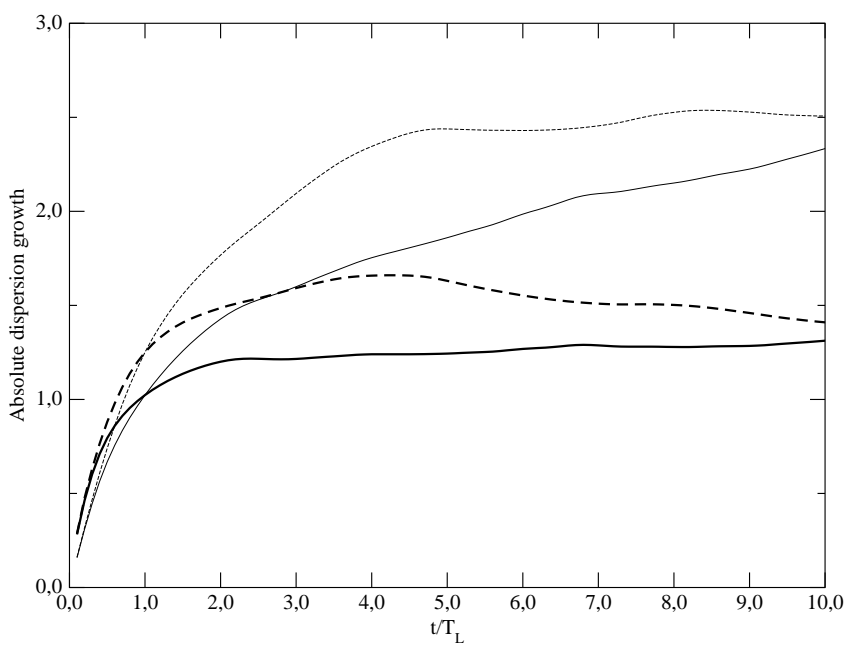

Fig. 6. Absolute dispersion compensated by $t$ (fine lines) and by $t^{5 / 4}$ (bold lines): DNS (continuous lines) and KS (dashed lines).

ing coherent vortices. As a result, hyperbolic periphery of vortices is a region where a number of particles may reside for a long time in spite of the natural Lagrangian stirring which favours the particle transition from one topological domain to an other. The same scenario may be conceived for KS Lagrangian dynamics. However, the difference is that the hyperbolic regions where $Q>0$ clearly extend over a more reduced area around elliptic concentrations in the KS field than in the DNS one (Fig. 3). Consequently, trapping efficiency of hyperbolic regions is more important in the DNS dynamics. It is quite reasonable to assume that trapping events make slack times in the absolute dispersion.

The anomalous behaviour in $t^{5 / 4}$ appears as a Lagrangian signature of hyperbolic domains and has a fundamentally important significance for the recognition of the Eulerian features from dispersion experiments. The robustness of the subdiffusion regime in $t^{5 / 4}$ disappears as soon as the action of the 2-D space-time structure is not present in the KS Lagrangian stirring.

\subsection{Relative dispersion}

Firstly, we will illustrate as in Zouari and Babiano (1994) how Richardson's scale-regime (19) may be observed in DNS for different developments of the inverse energy cascade. Later, we will compare DNS and KS relative dispersion in different numerical configurations.

The apparent insensitivity to the spectral energy distribution is analyzed on the basis of DNS fields R128F10 and R1024F256. The inverse energy cascade is absent in the first simulation even when a weak growth of the spectral energy density is observed at large scale. R1024F256 field exhibits a well developed inverse energy cascade where the theoretical prediction $k^{-5 / 3}$ is satisfied through one decade (see Fig. 1). 


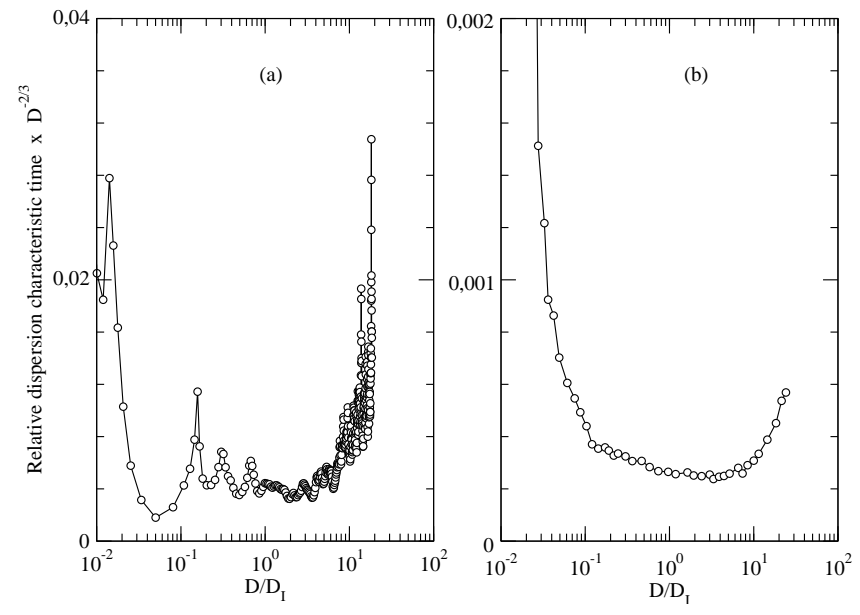

Fig. 7. Relative dispersion characteristic time $\tau_{R}$ compensated by $D^{2 / 3}$ as a function of the root-mean-square separation normalized by $D_{I}$. DNS experiments: (a) R128F10, (b) R1024F256.

The relative prominence of large-scale coherent vortices yields to $\Re_{v}=0.05$ and 0.002 , respectively. Larger $\Re_{v}$ means a more structured turbulence at large length-scale. On the other hand, the relative importance of the initial pair's separation $D_{0}$ with respect to the characteristic vortex-size, $D_{I}$, will be measured by the ratio $q=D_{0} / D_{I}$. Separation ratios $q$ in Lagrangian experiments are small enough: $q=0.0025,0.025$ respectively for R128F10 and R1024F256. Thus, the prominence of the coherent structures is more important in DNS R128F10 field rather than in DNS R1024F256 one and the ratio $q$ is smaller in a factor 10 .

The testing of the Richardson's scale-regime is performed through the relative dispersion characteristic time $\tau_{R}$ defined as Eq. (21). It is displayed in Fig. 7, where it is compensated by $D^{2 / 3}=<D^{2}>^{1 / 3}$. From Eq. (22), the Richardson's regime should thus correspond to a flat plateau. According to the self-similarity prediction (19), Richardson's scale-regime $Y(D) \sim D^{\beta}$ with $\beta=4 / 3$ and the corresponding growth in $D^{2 / 3}$ for $\tau_{R}$ are only consistent with the spectral slope $m=5 / 3$. The results presented in Fig. 7 show that $\tau_{R}$ behaviors are in excellent agreement with the phenomenological prediction (22) even if only the field R1024F256 exhibits $m=5 / 3$ (see Fig. 1). This originates a complicated situation in order to interpret the Richardson's law in the exclusive framework of usual self-similarity arguments.

\subsubsection{Sensitivity of DNS and KS relative dispersion to the ratio $q$.}

We are particularly interested in how DNS and KS differences can modify the achievement of Richardson's law. We will explore this problem comparing KS Lagrangian experiments to DNS numerical analyses performed in Babiano et al. (1990) and Zouari and Babiano (1994). Among all DNS

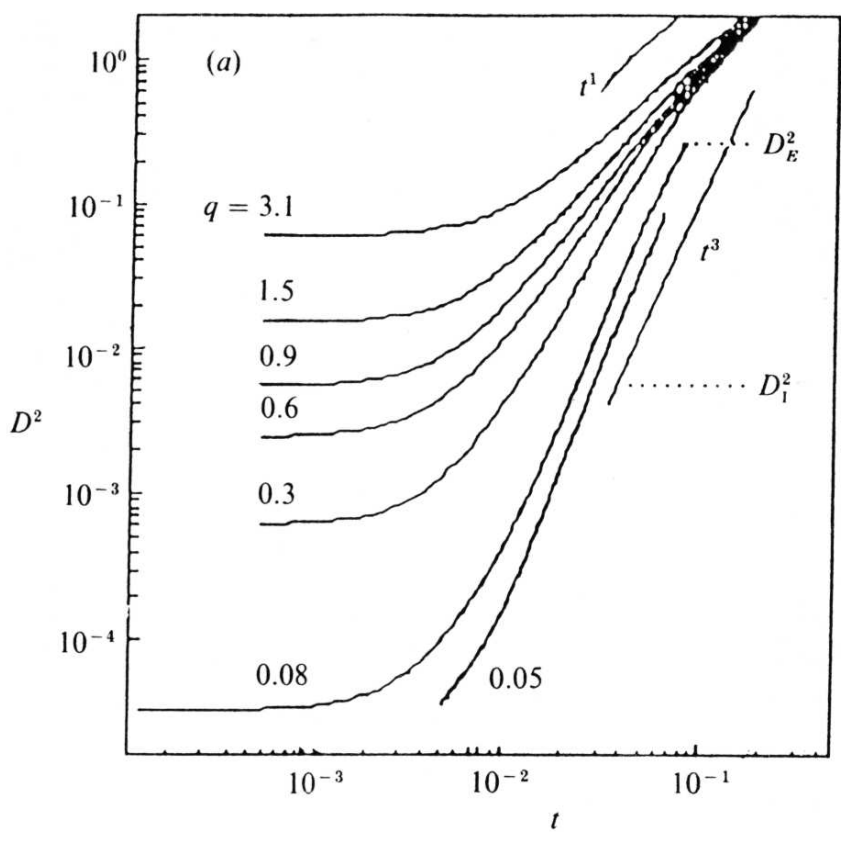

Fig. 8. Relative dispersion for DNS experiments, as a function of dispersion time, for different values of $q=D_{0} / D_{I}$. R256F40 and R1024F40 DNS experiments from Babiano et al. (1990).

fields analyzed in mentioned works, we will choose R256F40 and R512F40 where the dynamics is forced at wavenumber $k_{I}=40$ and the ratio $\Re_{v}$ is equal to 0.0125 . In terms of vortex prominence these fields represent an intermediate case compared to R128F10 and R1024F256 fields already analyzed in Fig. 7. They are characterized by dynamics which develops direct enstrophy and inverse energy cascades over a reasonable range of scales. The energy spectra for the R512F40 field have been presented in Fig. 1.

We have reproduced in Fig. 8 the study on relative dispersion $D^{2}$ for various values of $q$ previously published (Babiano et al., 1990). The energy inertial range $D_{I}^{2} \leq D^{2} \leq D_{E}^{2}$, where $D_{E}$ refers to the most energetic scale, is indicated. We observe that the growth of $D^{2}$ is significantly slower than the $t^{3}$ law (subdiffusion regime) when the initial separation is not small ( $q \geq 0.3$ ). Nevertheless, for $q \leq 0.3$ i.e., when $D_{0}$ lies at the bottom scales of the enstrophy range, the Richardson's times-regime in $t^{3}$ appears as a bound in the dispersion behaviour which seems to be quite robust and independent on $q$ in DNS Lagrangian experiments.

Figure 9 shows the relative dispersion $D^{2}$ in $\mathrm{KS} \mathrm{La-}$ grangian experiments performed on de basis of R512F40 and R256F40 fields. We observe in panel (a) that the Richardson's time-regime in $t^{3}$ is well verified for R512F40 DNS experiment ( $q=0.15$, circles) (Zouari and Babiano, 1994). This is consistent with the results presented in Fig. 8 for $q<0.3$. In contrast, the relative dispersion in $\mathrm{KS}$ experiment shows a slightly lower efficiency for an equivalent initial separation. However, for decreasing values of the initial 

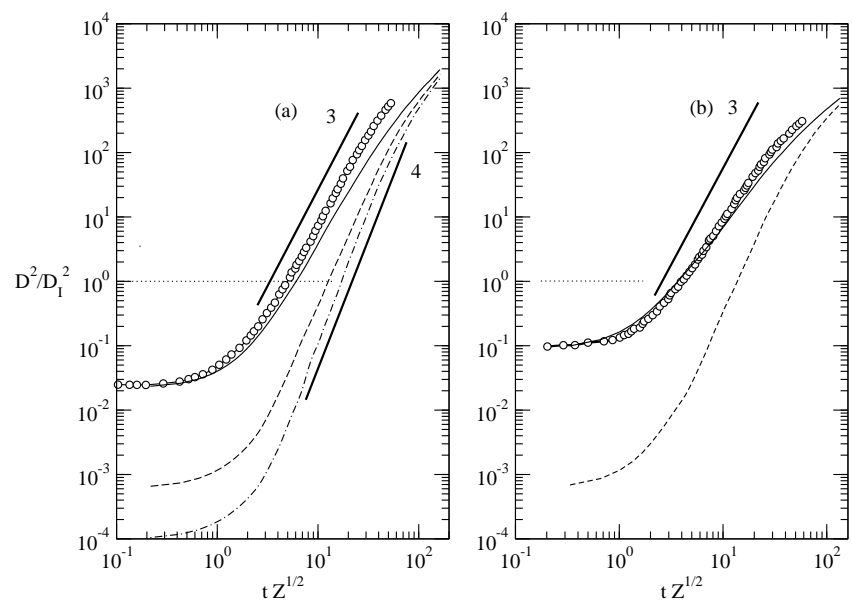

Fig. 9. Relative dispersion versus time in KS Lagrangian experiments for different values of $q=D_{0} / D_{I}$. Dispersion and time are normalized by $D_{I}^{2}$ and the Eulerian characteristic time $Z^{-1 / 2}$, respectively, where $Z$ is the enstrophy. (a) experiments R512F40: $q=0.15$ (continuous line), $q=0.025$ (dashed line), $q=0.01$ (dashedpoint line). (b) experiments R256F40: $q=0.3$ (continuous line), $q=0.025$ (dashed line). DNS experiment are also indicated for $\mathrm{R} 512 \mathrm{~F} 40, q=0.15$ in panel (a) and for R256F40, $q=0.3$ in panel (b) (circles).

separation $(q=0.025,0.01)$, hyperdiffusion regimes closed to $D^{2} \sim t^{4}$ are observed in KS relative dispersion behaviors in both R512F40 and R256F40 experiments. This is in contradiction with DNS behaviors in Fig. 8 which suggest a strong robustness of the $t^{3}$ regime for $q<0.3$. Results shown in Fig. 9 indicate that the $t^{3}$ law is replaced by an hyperdiffusion regime in $D^{2} \sim t^{4}$ in KS Lagrangian experiments for small enough values of $q$. Observed features in the relative dispersion are consistent with above analyzed properties of absolute dispersion processes: trapping events in vortices and well structured vortex-hyperbolic periphery produce a loss of time in the process of separation of particles pairs compared with an unstructured random separation motion.

Hyperdiffusion may be characterized by the relative dispersion characteristic time $\tau_{R}$, defined as Eq. (21). The behaviour of $\tau_{R}$ is presented in Fig. 10. In panel (a) it is shown as a function of the root-mean-square separation normalized as $D / D_{I}$. We compensated $\tau_{R}$ by its phenomenological scaling (Eq. 22) and we added a complementary DNS set with $q=0.025$ (triangles) in addition to previously commented DNS experiment with $q=0.15$ (circles). After having reached $D_{I}$ and, in spite of the difference in the initial pair's separations, both DNS relative dispersion characteristic times converge to an indistinguishable plateau which means that Richardson's scale-regime is reached in both cases. As $q$ decreases up to values which lie at the bottom of the enstrophy cascade (dashed lines), the identity in the behaviours of DNS and KS characteristic times ceases long before $D / D_{I} \approx 1$. At length-scales where the Richard-
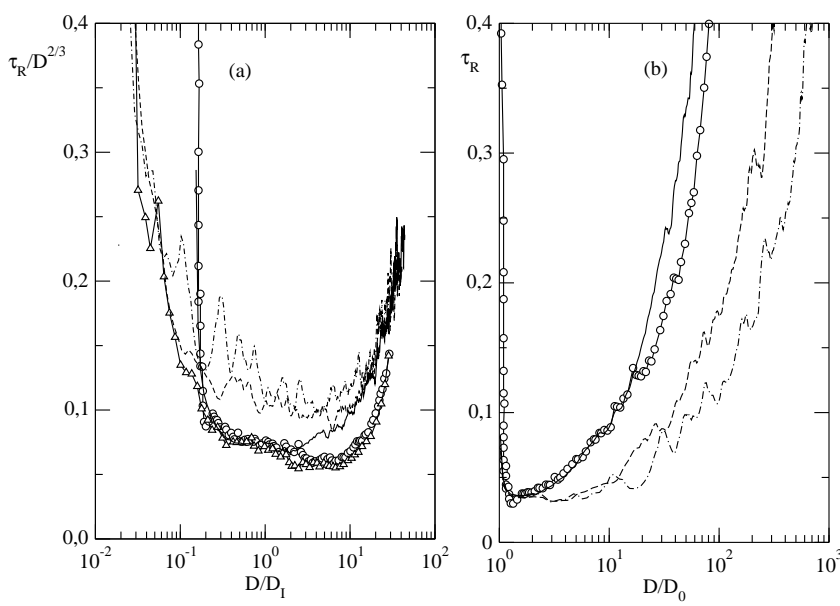

Fig. 10. (a) relative dispersion characteristic time, compensated by $D^{2 / 3}$, versus scale, normalized with forcing scale. (b) relative dispersion characteristic time versus scale, normalized with initial separation scale. DNS experiment R512F40: $q=0.15$ (circles), $q=0.025$ (triangles). KS experiments $q=0.15$ (continuous line), $q=0.025$ (dashed line), $q=0.01$ (dashed-point line).

son prediction is satisfied for DNS experiment, KS characteristic times are far from an horizontal plateau. Here the higher values of $\tau_{R}$ in KS experiments are not indicative of a less dispersion efficiency. In order to compare dispersion efficiencies, we plotted in panel (b) the characteristic dispersion times as a function of the relative dispersion normalized as $D / D_{0}$. For smaller values of $q$, KS characteristic dispersion times are smaller than DNS ones. This is consistent with the hyperdiffusion observed in the Fig. 9.

\subsubsection{Stationarity properties}

Stationarity properties of the above analyzed dispersion processes are investigated using the ratio $\Phi(t)$ and the integral $I(t)$, defined as Eqs. (32) and (30), respectively. We plot in Fig. 11a the ratio $\Phi$ as a function of the normalized dispersion time. We observe a decreasing function which, according to Eqs. (8), (13) and (31), must vanish as $\frac{d}{d t} D^{2}$ lobserved becomes constant for long dispersion times. When the Richardson's time-regime is observed in DNS relative dispersion (Fig. 9), $\Phi$ exhibits a plateau which means that there is a strong correlation between the $t^{3}$ growth and the saturation of the contributions of non-stationary terms in the exact Eq. (23). In KS experiments, $\Phi$ do not exhibit a perfect ceiling and show a monotonously decreasing behaviour which gives evidence to the fact that non-stationary contributions act for long dispersion times. Figure $11 \mathrm{~b}$ shows the dependence of the integral $I(t)$ as a function of time for various values of $q$ in KS experiments. The quasi-saturation of $I(t)$ is only attained for dispersion times of the order of $t>100 Z^{-1 / 2}$ when the KS relative dispersion already reaches its asymptotic regime (see Fig. 9). 

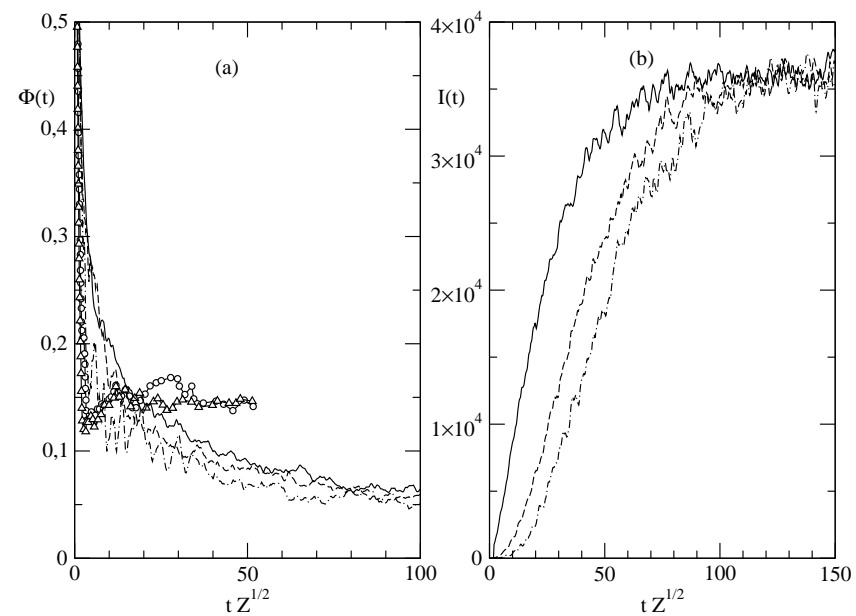

Fig. 11. (a) Stationarity criterion $\Phi$ defined as Eq. (32); (b) Integral $I(t)$, defined as (30). DNS experiments R512F40: $q=0.15$ (circles), $q=0.025$ (triangles). KS R512F40 experiments $q=0.15$ (continuous line), $q=0.025$ (dashed line), $q=0.01$ (dashed-point line).

We cannot compare DNS and KS $I(t)$-features for R512F40 field, since $I(t)$ behaviours have not been analyzed by Zouari and Babiano (1994). Nevertheless, we can perform the comparison for the R256F40 field and $q=0.025$. The correlations of relative accelerations $R_{\delta \Gamma}(t, \sigma)$, defined as Eq. (27), and $I(t)$ for this field are displayed in Fig. 12. Correlations $R_{\delta \Gamma}(t, \sigma)$ are plotted as a function of $\sigma Z^{1 / 2}$ at time $t$ when the stationarity is already attained. Integrals $I(t)$ are performed over $\sigma$ and plotted as a function of the dispersion time $t Z^{1 / 2}$. The integral $I(t)$ saturates at shorter times in DNS dispersion rather than in KS one. Approximately, there is a factor 4 between the two times for which we may roughly estimate that both integrals give a saturate behaviour. The saturation of $I(t)$ occurs for dispersion times $t Z^{1 / 2}$ larger than 15 in DNS Lagrangian experiment. In terms of nonnormalized dispersion time, this corresponds to $t=0.06$. The results shown in Fig. 8 for an equivalent value of $q$ indicate that the Richardson's regime is already robust enough at such dispersion times in DNS experiments. In the framework of the theoretical expressions (28-30), this regime corresponds to a constant in time behaviour of $I(t)$ as it is observed in Fig. 12b. In contrast, the integral $I(t)$ obviously shows a time-dependent behaviour all the range from 15 to 80 in KS Lagrangian experiment. The hyperdiffusion observed in Fig. $9 \mathrm{~b}$ at $q=0.025$ seems to be sustained by the quasi-linear in time growth of $I(t)$ before saturation occurs in the $\mathrm{KS}$ experiments.

All observed features are consistent with the theoretical expressions (28-30). Thus, the Richardson's $t^{3}$ dependency seems to be a saturate growth which is in fact the consequence of the saturation in the integral $I(t)$. When $I(t)$ does not saturate at intermediate dispersion time, then the dispersion process shows hyperdiffusion properties.

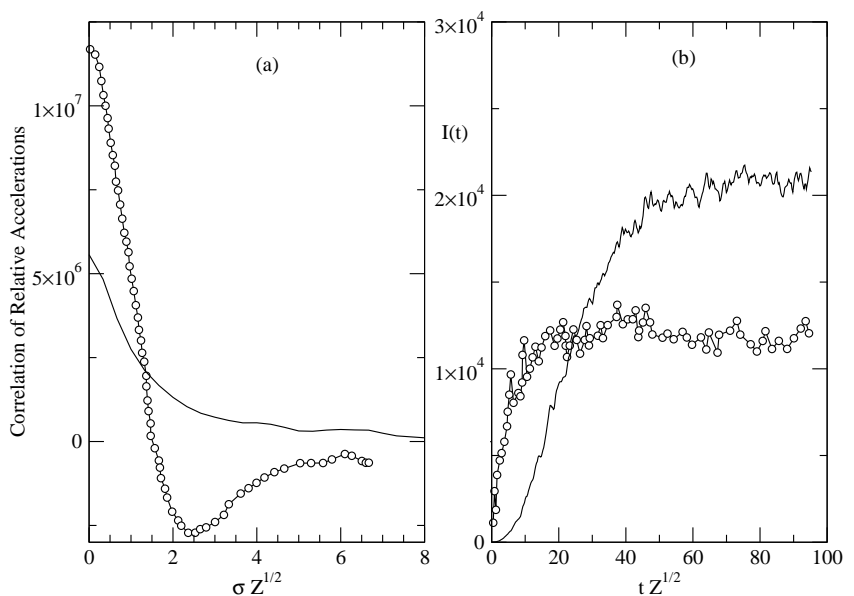

Fig. 12. (a) Correlation of relative accelerations, $R_{\delta \Gamma}$. (b) Integral $I(t)$, defined as Eq. (30). DNS (circles), KS (continuous line). R256F40 experiments and $q=0.025$

The second important difference between DNS and KS Lagrangian acceleration fields lies in $R_{\delta \Gamma}(t, \sigma)$ features (Fig. 12a). In KS, $R_{\delta \Gamma}(t, \sigma)$ is a positive defined function which monotonously decreases as $\sigma$ increases whereas in DNS $R_{\delta \Gamma}(t, \sigma)$ goes through zero at times of the order of the turnover time, $Z^{-1 / 2}$, and a significant anti-correlation is found. This is consistent with the fact that a lower $I(t)$-plateau is rapidly attained in DNS rather than in KS (Fig. 12b). The oscillation of $R_{\delta \Gamma}(t, \sigma)$ in DNS Lagrangian field can be interpreted as the mean signature of the relative movements of particle pairs around closed and long-lived coherent structures. Curves in Fig. 12a clearly show that the mean variance of relative acceleration $(\sigma=0)$ is rather higher in DNS fields than in KS one. This is presumably the consequence of quite different structure in the pressure fields. It is well known (Larchevêque, 1993) that in incompressible flow the Okubo-Weiss parameter $Q=\left(s^{2}-\omega^{2}\right) /\left(s^{2}+\omega^{2}\right)$, which defines the 2-D topology, is related to the Laplacian of pressure $P: Q=-4 \nabla^{2} P /\left(s^{2}+\omega^{2}\right)$.

\section{Conclusions and discussion}

In this work we have considered the comparison between DNS and KS Lagrangian experiments on turbulent particle dispersion in two-dimensional flows. We have noted that Eulerian structures of DNS and KS fields are not comparable, mostly due to the non-homogeneous space-time structure in DNS fields.

We have shown that the anomalous behaviour in $t^{5 / 4}$ for absolute dispersion at middle times discussed in Elhmaïdi et al. (1993) is unquestionably a consequence of the action of the 2-D structured topology. When the topology is closed to a random distribution in KS model then the behaviour in $t^{5 / 4}$ for the absolute dispersion is absent. 
The Richardson's law appears as quite robust behaviour for the relative dispersion in DNS Lagrangian experiments provided that the initial separation is small enough. Our interpretation is that the $t^{3}$ dependency for relative dispersion is a saturate growth which lies in the properties of the relative Lagrangian accelerations. It appears rather as the consequence of the ability of coherent structures to consolidate the stationarity regime in the relative acceleration field rather than the consequence of the inverse cascade of energy in twodimensional turbulence. Such an ability is significantly reduced in KS dynamics. In this case, the correlation of the relative particle accelerations reaches a stationarity regime only for times that are quite long compared to the time-span where the Richardson's law is already observed in DNS relative dispersion. Thus, KS Lagrangian dynamics generates an hyperdiffusion regime which lies in the fact that the integral $I(t)$, defined as Eq. (30), behaves as a time-dependent quantity for long times.

It is quite reasonable to assume that coherent vortices really contribute to the decorrelation of relative accelerations since, in large measure, they are responsible for the stirring and the curvature-change in Lagrangian trajectories. Our numerical results confirm this fact and suggest that such a decorrelation strongly reinforces the Richardson's regime. In the framework of the theory based on relations (23-30), this regime appears as a statistical bound in the growth of particle separations as soon as the integral $I(t)$ reaches a plateau.

From the physical standpoint, $I(t)$-plateau defines a characteristic Lagrangian time-scale for the Lagrangian relative acceleration field. By analogy with the usual Lagrangian definition (14), which characterize the absolute particle dispersion, we may define the characteristic Lagrangian timescale $T_{L \Gamma}$ which measures the decorrelation of the relative Lagrangian accelerations:

$T_{L \Gamma}=\lim _{t \rightarrow \infty} \Gamma_{0}^{-2} \int_{0}^{t}<\delta \boldsymbol{\Gamma}(t) . \delta \boldsymbol{\Gamma}(t-\sigma)>\mathrm{d} \sigma$,

where $\Gamma_{0}^{2}$ refers to the mean relative-acceleration variance along pair's-particle trajectories. We see that relative dispersion reduces in this case to two basic relations (28-30) which are exact under the assumption that a finite Lagrangian characteristic timescale $T_{L \Gamma}$ exists. Thus, the Richardson's regime follows from Eq. (29):

$G(t)=\frac{2}{3} \Gamma_{0}^{2} T_{L \Gamma} t$

It is possible to observe the parallelism between Eq. (40) and relations (12-13) which characterize absolute dispersion in the framework of Taylor-Kampé de Ferrier's theory. In both cases the working assumption of stationarity is necessary, however, Eq. (40) for relative dispersion is a transient behaviour at intermediate times whereas Eqs. (12-13) are really asymptotic.

We see that in the framework of the formulation (39-40) two questions are particularly important in order to estimate the relative particle dispersion. Firstly, the value of the finite Lagrangian characteristic timescale $T_{L \Gamma}$. Secondly, the dispersion time at which such a value is attained. If a quite short finite Lagrangian characteristic timescale $T_{L \Gamma}$ really exists, then the relative dispersion at intermediate times will be bounded by the Richardson's $t^{3}$ law. However, a wrong estimation of relative dispersion using Eqs. (28), (29) and (40) is conspicuous when $T_{L \Gamma}$ is sufficiently large compared to intermediate dispersion times. Nevertheless, within the limits of a reasonable tolerance, Eq. (29) may be useful to express sub-diffusion or hyper-diffusion regimes at intermediate dispersion times. The difference between the two regimes lies in the behaviour of $I(t)$ before saturation. According to our numerical observations, hyperdiffusion in $t^{4}$ can be approximated with a quasi-linear in time growth of $I(t)$.

Acknowledgements. Many thanks to the referees for their helpful suggestions and to C. Caulfield for his comments. R. Castilla was supported by an exchange grant in the framework of the European Science Foundation programme on Transport Processes in the Atmosphere and Ocean. European Union and Local grants ENVA4CT96-0334, ISTC-1481, FTN-2001, and the computing time of CESCA-CEPBA and the cluster of the Computing Service of U.P.C. in Terrassa are also acknowledged.

Edited by: A. Baas

Reviewed by: A. Provenzale and two other referees

\section{References}

Babiano, A., Basdevant, C., and Sadourny, R.: Structure functions and dispersion laws in two-dimensional turbulence, J. Atmos. Sci., 42, 942-949, 1985.

Babiano, A., Basdevant, C., Le Roy, P., and Sadourny, R... Single particle dispersion, Lagrangian structure function and Lagrangian energy spectrum in two-dimensional incompressible turbulence, J. Mar. Res., 45, 107-131, 1987.

Babiano, A. and Le Roy, P.: Quelques remarques sur la formulation non autosimilaire des lois de dispersion relative en turbulence bidimensionnelle, C.R. Acad. Sci. Paris, 305, II, 5-8, 1987.

Babiano,A., Basdevant, C., Le Roy, P., and Sadourny, R.: Relative dispersion in two-dimensional turbulence, J. Fluid Mech., 214, 535-557, 1990.

Batchelor, G. K.: Diffusion in fields of homogenous turbulence. II. The relative motion of particles, Proc. Camb. Phil. Soc., 48, 345362, 1952.

Bennet, A. F.: A Lagrangian analysis of turbulent diffusion, Rev. Geophys., 25, 799-822, 1987.

Castilla, R.: Simulación Cinemática de Flujo Turbulento. Aplicación al Estudio de la Estructura de la Turbulencia y de la Difusión Turbulenta, PhD. U.P.C., 2001.

Elhmaïdi, D., Babiano, A., and Provenzale, A.: Elementary topology of two-dimensional turbulence from Lagrangian viewpoint and single-particle dispersion, J. Fluid Mech., 257, 533-558, 1993.

Fung, J. C. H.: Kinematic simulation of turbulent flow and particle motion. PhD, University of Cambridge, 1990. 
Fung, J. C. H., Hunt, J. C. D., Malik, N. A., and Perkins, R. J.: Kinematic Simulation of homogeneous turbulence by unsteady random Fourier modes, J. Fluid Mech., 236, 281-318, 1992.

Fung, J. C. H. and Vassilicos, J. C.: Two-particle dispersion in turbulentlike flows, Phys. Rev. E, 52, 1677-1690, 1998.

Kampé de Fériet, J.: Les fonctions aléatoires stationaires et la théorie statistique de la turbulence homogène, Ann. Soc. Sci. Bruxelles, 59, 145-194, 1939.

Kraichnan, R. H.: Dispersion of particle pairs in homogeneous turbulence, Phys. Fluids, 9, 1937-1943, 1966.

Kraichnan, R. H.: Diffusion by a random velocity field, Phys. Fluids, 13(1), 22-31, 1970.

LaCasce J. and Bower A.: Relative dispersion in the subsurface North Atlantic, 2000, J. Mar. Res., 58, 863-894, 2000.

Larchevêque M. and Lesieur, M.: The application of eddy-damped Markovian closure to the problem of dispersion of particle pairs, J. Mecanique, 20, 113-134, 1981.

Larchevêque, M.: Pressure field and coherent structures in twodimensional incompressible flows, Theor. Comput. Fluid Dyn., 5, 215-222, 1993.

Lesieur, M. and Herring, J.: Diffusion of a passive scalar in twodimensional turbulence, J. Fluid Mech., 161, 77-95, 1985.

Lin, C. C. and Reid, W. H.: Turbulent flows: theoretical aspects, in: Handbuch der Physik, Vol. VIII/2, Springer, Berlin, 438-523, 1963.

Malik, A. M. and Vassilicos, J. C.: A Lagrangian model for turbulent dispersion with turbulent-like flow structure: Comparison with direct numerical simulation for two-particle statistics, Phys. Fluids, 11(6), 1572-1580, 1999.

McWilliams, J. C.: The emergence of isolated coherent structures in turbulent flow, J. Fluid Mech., 146, 21-43, 1984.

Monin, A. S. and Yaglom, A. M.: Statistical Fluid Mechanics, The MIT Press, 1975.
Obukhov, A. M.: Energy distribution in the spectrum of turbulent flow, Izv. Akad. Nauk. SSSR, Ser.-Geogr. i Geofiz., 5, 453-466, 1941.

Okubo, A.: Horizontal dispersion of floating particles in the vicinity of velocity singularities such as convergence, Deep-sea Res., 17, 445-454, 1970.

Ollitrault, M. and Colin de Verdière, A.: SOFAR Floats Reveal Midlatitude Intermediate North Atlantic General Circulation. Part I: A Lagrangian Descriptive View, J. Phys. Oceanogr., 32, 2020-2033, 2002.

Ollitrault, M. and Colin de Verdière, A.: SOFAR Floats Reveal Midlatitude Intermediate North Atlantic General Circulation. Part II: An Eulerian Statistical View, J. Phys. Oceanogr., 32, 2034-2053, 2002.

Pasquero, C., Provenzale, A., and Babiano, A.: Parameterization of dispersion in two-dimensional turbulence, Phys. Fluids, 439, 279-303, 2001.

Provenzale, A.: Transport by coherent barotropic vortices. Ann. Res. Fluid Mech., 31, 55-93, 1999.

Richardson, L. F.: Atmospheric diffusion shown in a distance neighbore graph, Proc. Roy. Soc. London A, 110, 709-737, 1926.

Sadourny, R. and Basdevant, C.: Parameterization of subgrid scale barotropic eddies in quasi-geostrophyc models: anticipated potential vorticity method, J. Atmos. Sci., 42, 1353-1363, 1985.

Taylor, G. I.: Diffusion by continuous movements, Proc. Lond. Math. Soc., 20, 196-212, 1921.

Weiss, J.: The dynamics of the enstrophy transfer in twodimensional turbulence, Physica D, 48, 273-294, 1991.

Zouari, N. and Babiano, A.: Derivation of the relative dispersion law in the inverse energy cascade of two-dimensional turbulence, Physica D, 76, 318-328, 1994. 